\title{
Stimulation of the Extracellular Matrix Production in Dermal Fibroblasts by Velvet Antler Extract
}

\author{
Seok-Seon Roh, O.M.D., Min-Ho Lee, M.S. ${ }^{1}$, Yul-Lye Hwang, M.S. ${ }^{1}$, Hyun-Hee Song, M.S. ${ }^{1}$, \\ Mu Hyun Jin, M.S. ${ }^{2}$, Sun Gyoo Park, M.S. ${ }^{2}$, Cheon Koo Lee, Pharm.D. ${ }^{2}$, Chang Deok Kim, Ph.D., \\ Tae-Jin Yoon, M.D. ${ }^{3}$, Jeung-Hoon Lee, M.D. \\ Department of Dermatology and the Research Institute for Medical Sciences, School of Medicine, Chungnam National University, Daejeon, \\ ${ }^{1}$ Oriental BioMed Lab, Daejeon, ${ }^{2}$ LG Household E Personal Care Ltd, Daejeon, ${ }^{3}$ Department of Dermatology and the Institute of Health \\ Sciences, School of Medicine, Gyeongsang National University, Jinju, Korea
}

Background: Fibroblasts produce many components of the extracellular matrix (ECM) and so they contribute to the maintenance of connective tissue integrity. Objective: The aim of this study is to evaluate the effect of velvet antler extract (VAE) on the ECM production of dermal fibroblasts cultured in vitro. Methods: Primary cultured human dermal fibroblasts were treated with VAE, and then the ECM production was determined by RT-PCR, ELISA and Western blot analysis. Furthermore, the change of gene expression according to VAE treatment was evaluated by cDNA microarray. Results: VAE accelerated the growth of fibroblasts in a dose-dependent manner. VAE increased the production of several ECM components, including type 1 collagen, fibronectin and elastin. In line with these results, the phosphorylations of p42/44 ERK and p38 mitogen-activated protein kinase were markedly increased by VAE, suggesting that the enhancement of ECM production may be linked to the activation of intracellular signaling cascades. VAE also significantly increased cell migration on an in vitro scratch wound test. In cDNA microarray, many genes related with connective tissue integrity were identified to be up-regulated by VAE. Conclusion: These results suggest that VAE has a potential to stimulate ECM production, and VAE may be

Received January 18, 2010, Revised February 11, 2010, Accepted for publication February 12, 2010

${ }^{*}$ This study was supported by a grant of the Korea Healthcare Technology R\&D Project, Ministry for Health, Welfare and Family Affairs, Republic of Korea (A080309). This study was also supported by a grant of the Korea Health 21 R\&D Project, Ministry of Health \& Welfare, Republic of Korea (01-PJ3-PG6-01GN12-0001).

Corresponding author: Jeung-Hoon Lee, M.D., Department of Dermatology, School of Medicine, Chungnam National University, 33 Munhwa-ro, Daejeon 301-040, Korea. Tel: 82-42-280-7707, Fax: 8242-280-8459, Email: jhoon@cnu.ac.kr applicable for maintaining the skin's texture. (Ann Dermatol 22(2) $173 \sim 179,2010$ )

-Keywords-

cDNA microarray, Extracellular matrix, Fibroblast, Velvet antler extract

\section{INTRODUCTION}

Fibroblasts are the major cells in the dermis, and these cells provide tensile strength and elasticity through the production and secretion of various components of the extracellular matrix (ECM), including collagens, proelastin, glycoproteins and proteoglycans ${ }^{1}$. Intracellular signaling cascades such as transforming growth factor- $\beta$ (TGF- $\beta$ ), extracellular signal-regulated kinases (ERKs), p38 mitogenactivated protein kinase (MAPK) and the $\mathrm{Wnt} \beta$-catenin pathway have been implicated in the regulation of ECM production in fibroblasts. With ageing, fibroblasts lose their proliferative potential and they show diminished ECM biosynthesis, resulting in dermal atrophy and wrinkle formation $^{2}$. In addition to their role for maintaining the mechanical properties of skin, fibroblasts also play a pivotal role during the wound healing process. Wound healing is a complex process that involves inflammation, granulation tissue formation and tissue remodeling, and this is all regulated by a number of cytokines and growth factors $^{3}$. During this process, fibroblasts proliferate and produce many of the ECM molecules in a TGF- $\beta$ dependent manner, and this contributes to the recovery of connective tissue integrity ${ }^{4}$. Thus, it has long been believed that enhancing the activity of fibroblasts, in the context of 
ECM production, may have beneficial effects on maintenance of skin texture.

Velvet antler is one of the most famous Korean traditional medicines, and it has been considered to contain some functional components for health promotion. It has been used for the treatment of bone disease, liver damage and anemia $^{5}$. Several studies have indicated that velvet antler extract (VAE) has immunosuppressive activity, an inhibitory potential for bone resorption and stimulatory effects on hematopoietic stem cells ${ }^{6-8}$. Although the diverse activities of VAE have been implicated in various biological systems, the potential effects of VAE on the skin have not yet been precisely determined. At present, there is only limited data available to support the potential effects of VAE on skin cells. For instance, a water extract and the polypeptides of velvet antler have a stimulatory effect on the proliferation of epidermal cells ${ }^{9}$, and topical treatment with VAE accelerated the repair of cutaneous wounds in diabetic rats ${ }^{10}$.

In the present study, we demonstrate that VAE stimulates the ECM production in dermal fibroblasts cultured in vitro, and that many genes related to connective tissue integrity were up-regulated by VAE.

\section{MATERIALS AND METHODS}

\section{Cell culture}

Normal human skin samples were obtained from circumcisions, in accordance with the ethical committee approval process of Chungnam National University Hospital. The specimens were briefly sterilized in $70 \%$ ethanol, minced and then incubated in DMEM supplemented with 10\% FBS and antibiotics (Gibco BRL, Rockville, MD, USA). Dermal fibroblasts normally outgrew from the explants after 5 7 days. At confluence, the cells were routinely passaged using a $1: 4$ split ratio and were used between passages 4 and 16 . For treatment with $\mathrm{VAE}$, approximately $1 \times 10^{6}$ cells were seeded on $100-\mathrm{mm}$ culture dishes and grown to sub-confluence. The cells were starved of serum for $24 \mathrm{~h}$, and then treated with VAE in serum-free medium.

\section{Preparation of VAE}

The dried velvet antler was crushed and extracted with cold ethanol. The ethanol-extract was concentrated in a vacuum evaporator (Buchi, Switzerland) and the resulting residue was weighed and dissolved to a $1 \%$ solution in $70 \%$ ethanol.

\section{Cell growth analysis}

For the $\left[{ }^{3} \mathrm{H}\right]$ thymidine uptake assay, fibroblasts were seeded in a 60-mm culture dish and treated with $1 \mu \mathrm{Ci}$ of $\left[{ }^{3} \mathrm{H}\right]$ thymidine (Amersham, Buckinghamshire, UK). Following incubation for the indicated time points, the cells were washed twice with PBS and incubated with $0.1 \mathrm{~N} \mathrm{NaOH}$ at room temperature. The radioactivity in the cell lysates was measured using a liquid scintillation counter.

\section{ELISA}

The ELISA kit for type 1 procollagen was purchased from Takara Bio Inc. (Shiga, Japan), and the ELISA kit for fibronectin was purchased from American Diagnostica (Greenwich, CT, USA). The levels of type 1 procollagen and fibronectin secreted from the fibroblasts were quantified according to the manufacturer's recommended protocols. The measurements were repeated at least 3 times, with independent cell batches.

\section{Western blot analysis}

The cells were lysed in Proprep solution (Intron, Daejeon, Korea). After vigorous pipetting, the extracts were centrifuged for $15 \mathrm{~min}$ at 13,000 rpm. The total protein was measured using a Bradford protein assay kit (Bio-Rad Laboratories, Hercules, CA, USA). The samples were run on SDS-polyacrylamide gels and transferred onto nitrocellulose membranes and then the proteins on the membranes were incubated with the appropriate antibodies for overnight at $4^{\circ} \mathrm{C}$ with gentle agitation. The blots were then incubated with the peroxidase-conjugated secondary antibodies for 30 minutes at room temperature, and visualized by enhanced chemiluminescence (Intron, Daejeon, Korea). The following primary antibodies were used in this study: collagen type $1 \alpha 1$ and elastin (Santa Cruz Biotechnologies, Santa Cruz, CA, USA), phospho-p38 MAPK, total-p38 MAPK, phospho-p42/44 ERK and totalp42/44 ERK (Cell Signaling Technology, Danvers, MA, USA) and actin (Sigma, St. Louis, MO, USA).

\section{In vitro wound healing assay}

When a new artificial gap is created on a confluent cell monolayer, the cells on the edge of the gap move toward the opening to close the gap until new cell-cell contacts are again established ${ }^{11}$. We performed an in vitro wound healing assay to evaluate the migration potency of the fibroblasts. Fibroblasts were grown to confluency and they were incubated with $10 \mu \mathrm{g} / \mathrm{ml}$ mitomycin C (Sigma) for 2 $\mathrm{h}$ to rule out the proliferative effect. A cell-free area was introduced by scraping the monolayer with a pipette tip. After incubation, the cells were photographed by using an inverted phase-contrast microscope. 
Table 1. Nucleotide sequence of the primers

\begin{tabular}{|c|c|c|c|}
\hline Name & Primer & & Expected size $(b p)$ \\
\hline \multirow[t]{2}{*}{ TGF- $\beta 1$} & Forward $\left(5^{\prime} \rightarrow 3^{\prime}\right)$ & CATCAACGGGTTCACTACCG & 431 \\
\hline & Reverse $\left(5^{\prime} \rightarrow 3^{\prime}\right)$ & CCACGTAGTACACGATGGGC & \\
\hline \multirow[t]{2}{*}{ FGF-19 } & Forward $\left(5^{\prime} \rightarrow 3^{\prime}\right)$ & AACCCCATGTGGGAATTGAT & 419 \\
\hline & Reverse $\left(5^{\prime} \rightarrow 3^{\prime}\right)$ & GCTGCTTCCACACAGCAAGT & \\
\hline \multirow[t]{2}{*}{ FGF-1 } & Forward $\left(5^{\prime} \rightarrow 3^{\prime}\right)$ & AGCCCACAGAGCCTGAATTT & 339 \\
\hline & Reverse $\left(5^{\prime} \rightarrow 3^{\prime}\right)$ & CAGGAAGGACAAAAGGGAGC & \\
\hline \multirow[t]{2}{*}{ IGF-2 } & Forward $\left(5^{\prime} \rightarrow 3^{\prime}\right)$ & САСССТCСАGTTCGTCTGTG & 398 \\
\hline & Reverse $\left(5^{\prime} \rightarrow 3^{\prime}\right)$ & TTGGGTGGGTAGAGCAATCA & \\
\hline \multirow[t]{2}{*}{ Collagen type 3} & Forward $\left(5^{\prime} \rightarrow 3^{\prime}\right)$ & TCATGCССТАСТGGTCСТCA & 301 \\
\hline & Reverse $\left(5^{\prime} \rightarrow 3^{\prime}\right)$ & GTCGTCCGGGTCTACCTGAT & \\
\hline \multirow[t]{2}{*}{ FLT-1 } & Forward $\left(5^{\prime} \rightarrow 3^{\prime}\right)$ & GCCСАTACTTTTGGСТССТC & 455 \\
\hline & Reverse $\left(5^{\prime} \rightarrow 3^{\prime}\right)$ & TATGCGCCAGCTAATGCTCT & \\
\hline \multirow[t]{2}{*}{ Actin } & Forward $\left(5^{\prime} \rightarrow 3^{\prime}\right)$ & AAACTGGAACGGTGAAGGTG & 352 \\
\hline & Reverse $\left(5^{\prime} \rightarrow 3^{\prime}\right)$ & CTCAAGTTGGGGGACAAAAA & \\
\hline
\end{tabular}

\section{Total RNA extraction and cDNA microarray analysis}

Total RNA was extracted from the cells using the Trizol Reagent Kit (Invitrogen) according to the manufacturer's instructions. For the microarray analysis, a $22 \mathrm{~K}$ Human Genome Array was used (Genomictree, Daejeon, Korea). One hundred $\mu \mathrm{g}$ total RNA was reverse transcribed in the presence of Cy3 and/or Cy5 monoreactive dye (Amersham, Little Chalfont, UK) using a Superscript cDNA synthesis system (Gibco BRL, Rockville, MD, USA). After hybridization, microarray scanning and data normalization were performed using a GenePix 4000B scanner and GenePix Pro 3.0 software (Axon Instruments, Union City, CA, USA). The differentially expressed genes were selected based on the fold-change and Welch's t-test.

\section{RT-PCR}

Two $\mu \mathrm{g}$ of total RNA were reverse-transcribed using M-MLV reverse transcriptase (ELPIS Biotech, Daejeon, Korea). Aliquots of the RT mixture were subjected to PCR with specific primer sets (Table 1).

\section{RESULTS}

It has been previously reported that the polypeptides from velvet antler have a stimulatory effect on the proliferation of epidermal cells ${ }^{9}$. To investigate whether VAE has a potential for stimulating cell growth, we first performed a $\left[{ }^{3} \mathrm{H}\right]$ thymidine uptake assay. Dermal fibroblasts were serially treated with various concentrations of VAE and incubated for 2 days. Consistent with the previous reports, VAE increased the $\left[{ }^{3} \mathrm{H}\right]$ thymidine uptake of the fibroblasts in a dose-dependent manner (Fig. 1). Since the proliferative property of fibroblasts is frequently linked to the ECM production capability, we next determined the effect of VAE on the production of several ECM components. ELISA

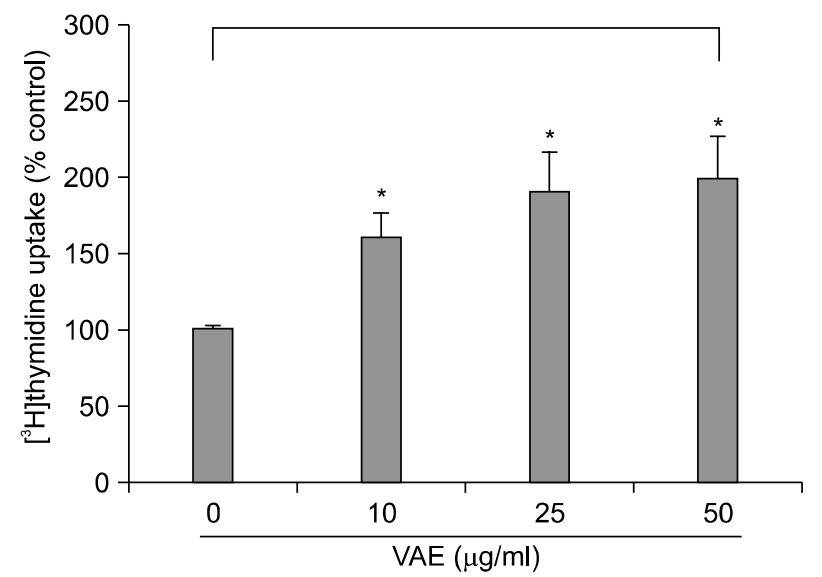

Fig. 1. Effect of velvet antler extract (VAE) on the growth of dermal fibroblasts. The cells were treated with VAE at the indicated concentrations for 2 days in the presence of $\left[{ }^{3} \mathrm{H}\right]$ thymidine. The radioactivity was measured by a liquid scintillation counter. The results are shown as percentage of the control \pm standard deviation (SD) $\left({ }^{*} p<0.05\right.$ vs. control).

assay showed that VAE increased the secretion of type 1 procollagen and fibronectin in a dose-dependent manner (Fig. 2A, B). Concomitantly, Western blotting demonstrated that VAE increased the intracellular protein levels of collagen type $1 \alpha 1$ and other connective tissue components of elastin (Fig. 2C).

As the intracellular signaling cascades such as p42/44 ERK and p38 MAPK cascades have been implicated in the regulation of ECM production ${ }^{12,13}$, we investigated the effect of VAE on MAPK activation. As shown in Fig. 3, VAE treatment led to quick phosphorylation of $\mathrm{p} 42 / 44$ ERK and p38 MAPK. The phosphorylation then gradually diminished in a time-dependent manner. These results suggest that VAE affects the intracellular signaling cascades and thereby has an influence on the expression of ECM components. 
A

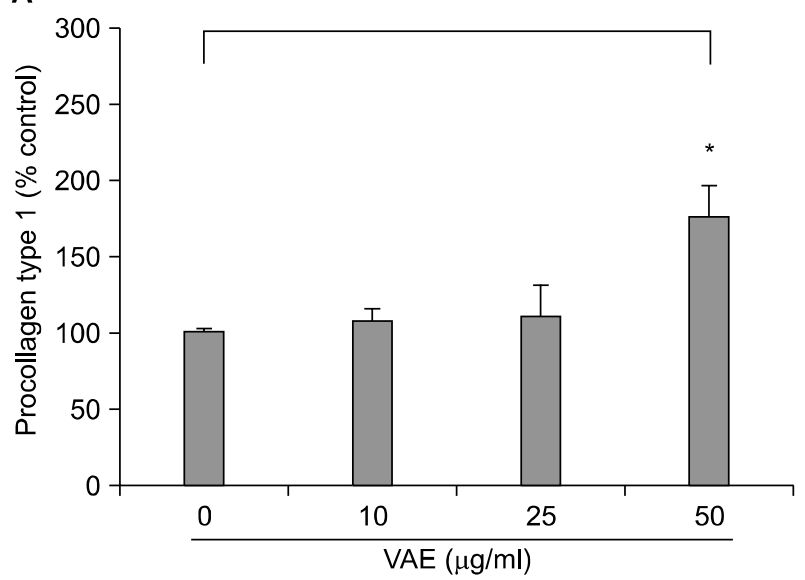

C
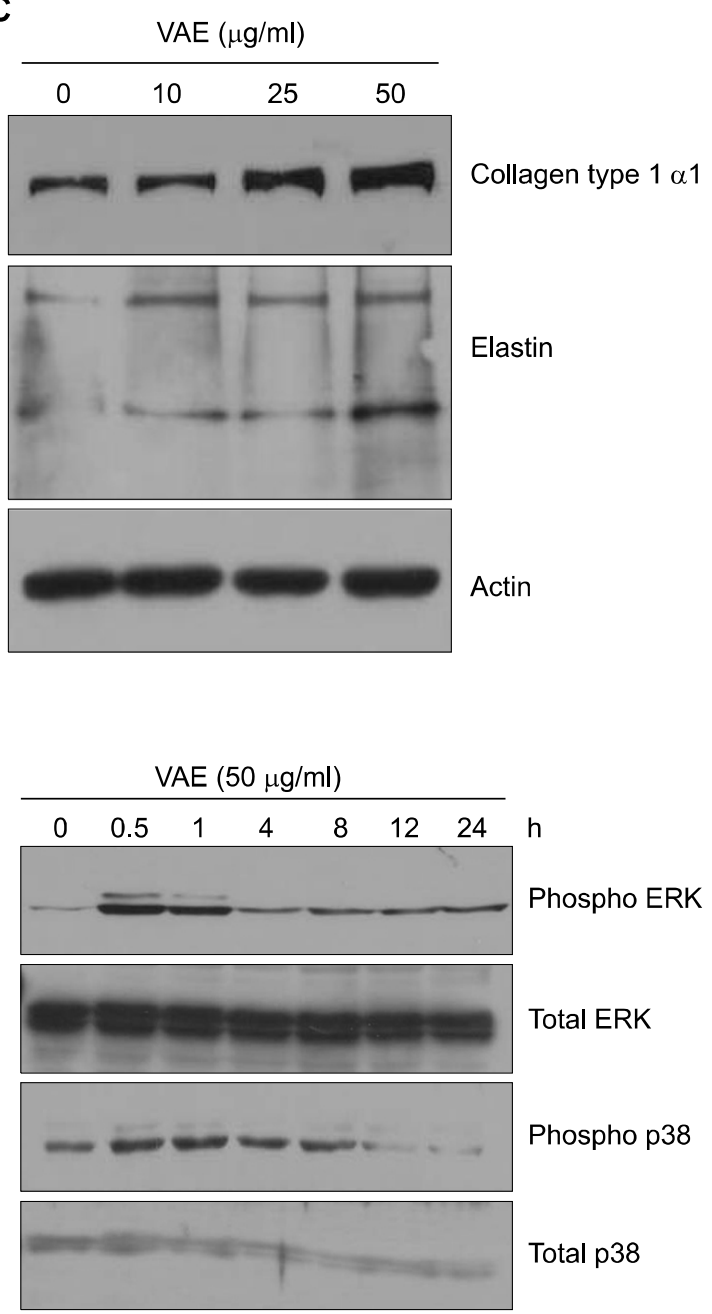

Fig. 3. Effect of velvet antler extract (VAE) on intracellular signaling pathway. The cells were treated with VAE for the indicated time points. The cellular proteins were prepared and the phosphorylations of ERK and p38 MAPK were determined by Western blot analysis.
B

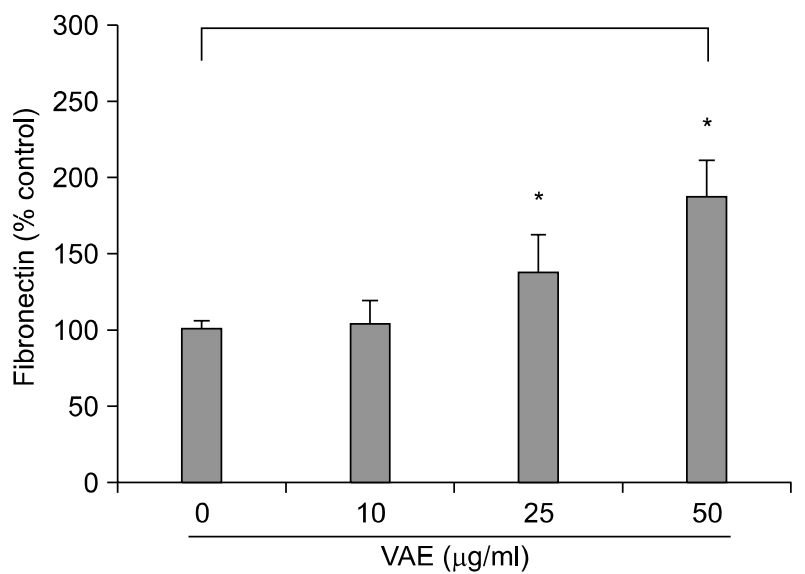

Fig. 2. Effect of velvet antler extract (VAE) on the extracellular matrix (ECM) production of dermal fibroblasts. The cells were treated with VAE at the indicated concentrations for 2 days. The conditioned medium was collected, and the secreted procollagen type 1 (A) and fibronectin (B) were measured by ELISA. The results are shown as percentage of the control \pm standard deviation (SD) $(* p<0.05$ vs. control). (C) The cellular proteins were harvested and the protein levels for collagen type $1 \alpha 1$ and elastin were verified by Western blot analysis.

Previous data indicates that VAE accelerated the wound healing in diabetic rats $^{10}$. During the wound closure, fibroblasts extensively proliferate and migrate, and then they fill the wound gap. Thus, we next determined whether VAE has a potential for enhancing cell migration. We carried out an in vitro scratch assay because it is considered to be a straightforward and economical method to study cell migration. Faster closure of the scratch wound was observed in the VAE-treated group as compared with that of the control group (Fig. 4).

We carried out cDNA microarray analysis to further delineate the effects of VAE on fibroblasts. As a result, we obtained about 1,200 differentially expressed genes by VAE treatment. We selected several genes that may be linked to the wound healing process and that showed their fold inductions in cDNA microarray (Table 2). We then performed RT-PCR analysis to confirm the cDNA microarray results. The expression levels for TGF- $\beta 1$, FGF-19, FGF-1, IGF-2, collagen type $3 \alpha 1$ and FLT-1 were significantly increased by VAE in a dose-dependent 


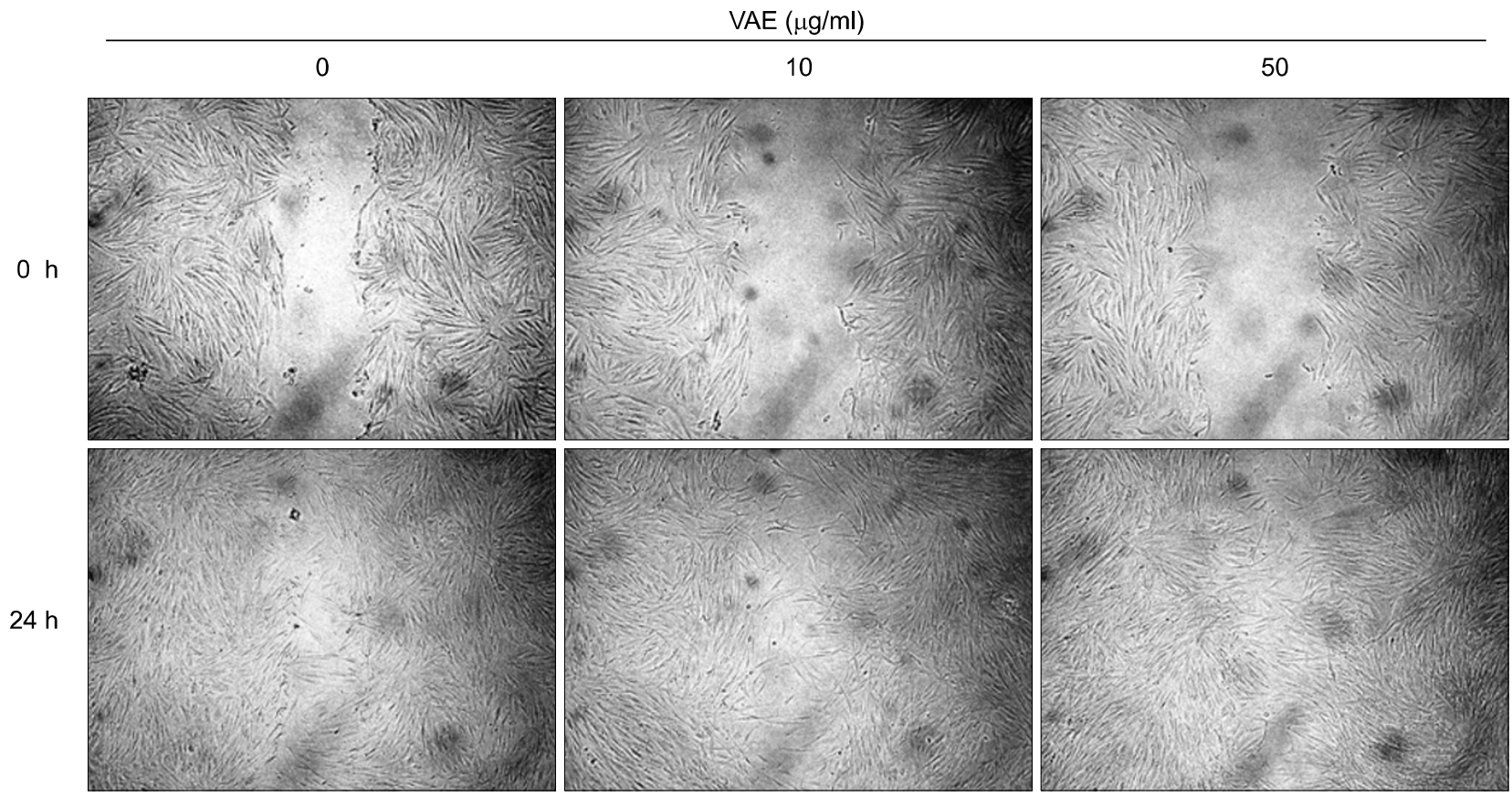

Fig. 4. Effect of velvet antler extract (VAE) on cell migration. Confluent monolayers of dermal fibroblasts were wounded using a pipette tip, and the monolayer cells were treated with VAE at the indicated concentrations. The areas of cell-free wounds were photographed after $24 \mathrm{~h}$ treatment.

Table 2. Representative genes that are highly up-regulated by VAE in fibroblasts

\begin{tabular}{lllr}
\hline Accession No. & \multicolumn{1}{c}{ Gene name } & Abbreviation & Fold induction* \\
\hline NM_000660 & transforming growth factor, beta 1 & TGF- $\beta 1$ & 10.78 \\
NM_005117 & fibroblast growth factor 19 & FGF-19 & 64.23 \\
NM_000800 & fibroblast growth factor 1 & FGF-1 & 5.72 \\
NM_000612 & insulin-like growth factor 2 & IGF-2 & 4.53 \\
NM_000090 & collagen, type III, alpha 1 & COL3A1 & 4.12 \\
NM_002019 & fms-related tyrosine kinase 1 & FLT-1 & 8.99 \\
\hline
\end{tabular}

*Dermal fibroblasts were treated with $50 \mu \mathrm{g} / \mathrm{ml}$ velvet antler extract (VAE) for 2 days. The total RNAs were isolated and used for preparation of the Cy3- or Cy5-labeled probes.

manner (Fig. 5), which well-matched the results of the cDNA microarray.

\section{DISCUSSION}

The maintenance of skin texture in the context of mechanical properties, including tensile strength and elasticity, is largely dependent on the activities of fibroblasts. This notion is clearly supported by the fact that biosynthesis of the ECM by fibroblasts is markedly diminished in aged persons ${ }^{2}$. Therefore, the approach that enhances ECM production and thereby strengthens the skin texture is an attractive method for curing connective tissue-related skin phenotypes such as wrinkle formation. In this study, we attempted to validate the potential effect of VAE on ECM production by using primary cultured dermal fibroblasts.
We demonstrated that $\mathrm{VAE}$, a frequently used traditional medicine, has a potential for enhancing fibroblasts' proliferation and cell migration, and also that VAE increases the production of fibronectin, type 1 collagen and elastin. In addition, the cDNA microarray showed that several growth factors were greatly increased by VAE treatment of fibroblasts. Fibronectin, type 1 collagen and elastin are important constituents in connective tissue. Fibronectin is a multifunctional glycoprotein that is involved in the interaction between fibroblasts and the ECM. It is secreted from various cells, including fibroblasts, in the early stage of wound healing, and fibronectin induces cell migration, suggesting that it plays a critical role in the wound healing process ${ }^{14}$. Type I collagen plays a critical role in maintenance of the tensile strength of skin, and the dermis predominantly contains type 1 collagen, which is synthe- 


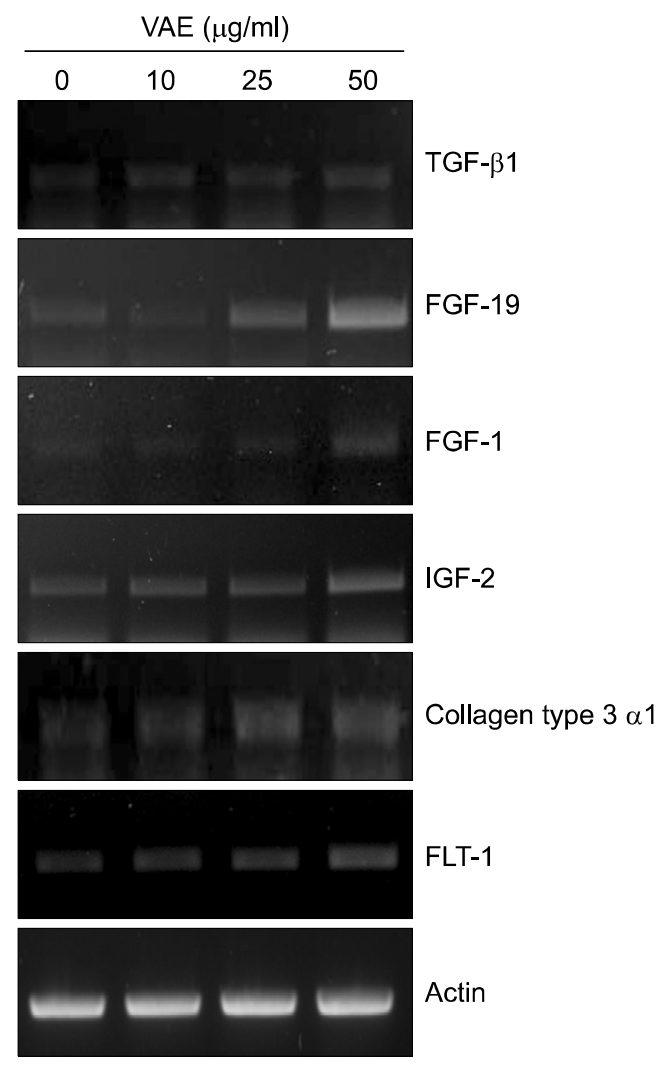

Fig. 5. Dermal fibroblasts were treated with velvet antler extract (VAE) at the indicated concentrations for 2 days. The levels of expression of selected genes were verified by RT-PCR.

sized from dermal fibroblasts as precursor molecules called procollagen ${ }^{15}$. Elastin is physiologically important for the elasticity of skin, and it is also secreted from skin fibroblasts as a soluble precursor tropoelastin, which is subsequently cross-linked into insoluble elastin ${ }^{16}$. Based on the potential effect of VAE on those ECM components, it is expected that VAE can be used for strengthening of skin texture and/or curing of connective tissue-related skin phenotypes.

Interestingly, the effects of VAE on fibroblasts are somewhat similar to what can be seen in the wound healing process; cell proliferation, migration and ECM production. It has been well established that several growth factors, including TGF- $\beta 1$, IGF-2 and platelet-derived growth factor, are up-regulated during the growth phase of cutaneous repair $^{17}$. Among them, TGF- $\beta$ is recognized as the most important modulator that evokes activation of the Smad signaling cascades. In our study, VAE treatment resulted in significant increases of TGF- $\beta 1$, together with several growth factors such as FGF-19, FGF-1 and IGF-2. Furthermore, we found that the expression of FLT-1 (fms-like tyrosine kinase) was up-regulated by VAE. The FLT-1 is a receptor for vascular endothelial growth factor
$(\mathrm{VEGF})^{18}$, which participates in angiogenesis. Taken together, our data suggest that VAE may lead the fibroblasts towards the activated status that can be seen in the wound healing process, and this raises the possibility of therapeutically using VAE for fast wound closure.

In summary, we demonstrated that VAE has a potential for stimulating ECM production in dermal fibroblasts. Since the effect of VAE on the ECM production in fibroblasts had not been previously investigated, our data provides novel clues on which to base further investigations on the potential applications of VAE. Our results suggest that VAE could be used for connective tissue-related skin phenotypes as a co-modality together with first-line treatments.

\section{REFERENCES}

1. Pieraggi MT, Bouissou H, Angelier C, Uhart D, Magnol JP, Kokolo J. The fibroblast. Ann Pathol 1985;5:65-76.

2. Uitto J. Connective tissue biochemistry of the aging dermis. Age-related alterations in collagen and elastin. Dermatol Clin 1986;4:433-446.

3. Chan JC, Duszczyszyn DA, Castellino FJ, Ploplis VA. Accelerated skin wound healing in plasminogen activator inhibitor-1-deficient mice. Am J Pathol 2001;159:1681-1688.

4. Ignotz RA, Massague J. Transforming growth factor-beta stimulates the expression of fibronectin and collagen and their incorporation into the extracellular matrix. J Biol Chem 1986;261:4337-4345.

5. Jhon GJ, Park SY, Han SY, Lee S, Kim Y, Chang YS. Studies of the chemical structure of gangliosides in deer antler, Cervus nippon. Chem Pharm Bull (Tokyo) 1999;47:123-127.

6. Kang SK, Kim KS, Kim SI, Chung $\mathrm{KH}$, Lee IS, Kim $\mathrm{CH}$. Immunosuppressive activity of deer antler extracts of Cervus korean TEMMINCK var. mantchuricus Swinhoe, on type II collagen-induced arthritis. In Vitro Cell Dev Biol Anim 2006;42:100-107.

7. Li YJ, Kim TH, Kwak HB, Lee ZH, Lee SY, Jhon GJ. Chloroform extract of deer antler inhibits osteoclast differentiation and bone resorption. J Ethnopharmacol 2007;113: 191-198.

8. Yang HO, Kim SH, Cho SH, Kim MG, Seo JY, Park JS, et al. Purification and structural determination of hematopoietic stem cell-stimulating monoacetyldiglycerides from Cervus nippon (deer antler). Chem Pharm Bull (Tokyo) 2004;52: 874-878.

9. Guan SW, Duan LX, Li YY, Wang BX, Zhou QL. A novel polypeptide from Cervus nippon Temminck proliferation of epidermal cells and NIH3T3 cell line. Acta Biochim Pol 2006;53:395-397.

10. Mikler JR, Theoret CL, High JC. Effects of topical elk velvet antler on cutaneous wound healing in streptozotocininduced diabetic rats. J Altern Complement Med 2004;10: 835-840.

11. Liang CC, Park AY, Guan JL. In vitro scratch assay: a convenient and inexpensive method for analysis of cell 
migration in vitro. Nat Protoc 2007;2:329-333.

12. Lim IJ, Phan TT, Tan EK, Nguyen TT, Tran E, Longaker MT, et al. Synchronous activation of ERK and phosphatidylinositol 3-kinase pathways is required for collagen and extracellular matrix production in keloids. J Biol Chem 2003;278:40851-40858.

13. Lee DJ, Rosenfeldt H, Grinnell F. Activation of ERK and p38 MAP kinases in human fibroblasts during collagen matrix contraction. Exp Cell Res 2000;257:190-197.

14. Kanzaki T, Morisaki N, Shiina R, Saito Y. Role of transforming growth factor-beta pathway in the mechanism of wound healing by saponin from Ginseng Radix rubra. Br J Pharmacol 1998;125:255-262.
15. Quan T, He T, Kang S, Voorhees JJ, Fisher GJ. Solar ultraviolet irradiation reduces collagen in photoaged human skin by blocking transforming growth factor-beta type II receptor/Smad signaling. Am J Pathol 2004;165:741-751.

16. Sephel GC, Davidson JM. Elastin production in human skin fibroblast cultures and its decline with age. J Invest Dermatol 1986;86:279-285.

17. Falanga $V$, Isaacs $C$, Paquette $D$, Downing $G$, Kouttab N, Butmarc J, et al. Wounding of bioengineered skin: cellular and molecular aspects after injury. J Invest Dermatol 2002;119:653-660.

18. Ferrara N, Davis-Smyth T. The biology of vascular endothelial growth factor. Endocr Rev 1997;18:4-25. 